\title{
Streptococcus pneumoniae Serotype 14 IgG Antibody Measurement
}

National Cancer Institute

\section{Source}

National Cancer Institute. Streptococcus pneumoniae Serotype 14 Ig G Antibody

Measurement. NCI Thesaurus. Code C142262.

The determination of the amount of Streptococcus pneumoniae serotype 14 Ig G antibody present in a sample. 